\title{
Direct Activation of Rat Spinal Dorsal Horn Neurons by Prostaglandin E2
}

\author{
Hiroshi Baba, Tatsuro Kohno, Kimberly A. Moore, and Clifford J. Woolf \\ Neural Plasticity Research Group, Department of Anesthesia and Critical Care, Massachusetts General Hospital and \\ Harvard Medical School, Charlestown, Massachusetts 02129
}

Whole-cell patch-clamp and intracellular recording techniques have been used to study the action of prostaglandin E2 (PGE2) on neurons in adult rat transverse spinal cord slices. Bathapplied PGE2 (1-20 $\mu \mathrm{m})$ induced an inward current or membrane depolarization in the majority of deep dorsal horn neurons (laminas III-VI; 83 of 139 cells), but only in a minority of lamina II neurons (6 of 53 cells). PGE2 alone never elicited spontaneous action potentials; however, it did convert subthreshold EPSPs to suprathreshold, leading to action potential generation. PGE2-induced inward currents were unaffected by perfusion with either a $\mathrm{Ca}^{2+}$-free/high $\mathrm{Mg}^{2+}(5 \mathrm{~mm})$ solution or tetrodotoxin $(1 \mu \mathrm{M})$, indicating a direct postsynaptic action. Both 17-phenyl trinor prostaglandin E2 (an EP1 agonist) and sulprostone (an EP3 agonist) had little effect on membrane current, whereas butaprost methyl ester (an EP2 agonist) mim- icked the effect of PGE2. Depolarizing responses to PGE2 were associated with a decrease in input resistance, and the amplitude of inward current was decreased as the holding potential was depolarized. PGE2-induced inward currents were reduced by substitution of extracellular $\mathrm{Na}^{+}$with $\mathrm{N}$-methyl-D-glucamine and inhibited by flufenamic acid (50-200 $\mu \mathrm{M})$, which is compatible with activation of a nonselective cation channel. These results suggest that PGE2, acting via an EP2-like receptor, directly depolarizes spinal neurons. Moreover, these findings imply an involvement of spinal cord-generated prostanoids in modulating sensory processing through an alteration in dorsal horn neuronal excitability.

Key words: inflammation; central sensitization; prostaglandin E2; EP2 receptor; deep dorsal horn; allodynia; hyperalgesia
After peripheral tissue injury or inflammation, exaggerated pain behavior occurs that includes hyperalgesia, an increased responsiveness to noxious stimuli, and allodynia, pain elicited in response to normally innocuous stimuli. Prostaglandins (PGs) synthesized by the inducible isoform of cyclooxygenase (COX-2) at the site of tissue injury sensitize peripheral nociceptors through activation of EP receptors on peripheral nerve terminals (Kumazawa et al., 1993). This peripheral sensitizing action contributes to heightened sensitivity at the site of tissue injury (primary hyperalgesia) (Ohuchi et al., 1976; Baccaglini and Hogan, 1983). Therefore, the analgesic effects of COX inhibitors [i.e., nonsteroidal anti-inflammatory drugs (NSAIDs)] have long been thought to be mediated primarily through inhibition of PG generation in the periphery (Vinegar et al., 1976). Recently, however, evidence has accumulated to indicate that PGE2 is also produced in the spinal cord after tissue injury (Yang et al., 1996; Dirig and Yaksh, 1999; Samad et al., 2001). Furthermore, behavioral studies suggest that PGE2 may facilitate nociceptive transmission in the spinal cord (Uda et al., 1990; Minami et al., 1994), contributing to central sensitization, a central amplification of sensory outflow from the spinal cord that is responsible for the

Received March 31, 2000; revised Dec. 7, 2000; accepted Dec. 18, 2000.

This work was supported by Human Frontier Science Program Grant RG73/96 and National Institutes of Health Grant NS38253-01. Dr. Baba was also supported by the Ministry of Education, Science, Sports and Culture of Japan (Niigata University, Niigata, Japan). We thank Ono Pharmaceutical Co. (Osaka, Japan) for the gift of PGE2.

Correspondence should be addressed to Dr. Hiroshi Baba, Department of Anesthesiology, Niigata University School of Medicine, 1-757 Asahimachi, Niigata 9518510, Japan. E-mail: baba@med.niigata-u.ac.jp.

Copyright (C) 2001 Society for Neuroscience 0270-6474/01/211750-07\$15.00/0 spread of sensitivity beyond the site of injury (Woolf and King, 1990).

Constitutive levels of COX-2 in the spinal cord are low, but peripheral inflammation upregulates this enzyme (Beiche et al., 1996, 1998; Ichitani et al., 1997), leading to production of PGE2 in the spinal cord (Yang et al., 1996; Dirig and Yaksh, 1999). Intrathecally administered PGE2 evokes hyperalgesia and allodynia (Uda et al., 1990; Minami et al., 1994), and both the behavioral hyperalgesia and the increase in spinal PGE2 concentration induced by peripheral inflammation are attenuated by intrathecally administered NSAIDs (Malmberg and Yaksh, 1992, 1995; Yamamoto and Nozaki-Taguchi, 1997). Intrathecal NSAIDs also reduce noxious stimulus-induced or peripheral inflammation-induced dorsal horn neuron hyperexcitability (Chapman and Dickenson, 1992; Pitcher and Henry, 1999). Although these studies collectively suggest a nociceptive role for PGE2 produced by COX in the spinal cord, it is uncertain what is responsible for its production, where it is produced, and where and how it acts.

Autoradiographic studies indicate that the highest density of spinal PGE2 binding sites is in lamina II, substantia gelatinosa (SG). PGE2 binding in lamina II is reduced, but not eliminated, by dorsal rhizotomy (Matsumura et al., 1995), suggesting that receptor sites are located on both presynaptic terminals of unmyelinated nociceptive fibers and postsynaptic dorsal horn neurons. Therefore, PGE2 may act presynaptically to facilitate neurotransmitter release (Nicol et al., 1992; Hingtgen et al., 1995; Vasko, 1995) and postsynaptically to directly excite dorsal horn neurons. Here, we demonstrate that PGE2 does directly activate a subpopulation of spinal dorsal horn neurons. 
A

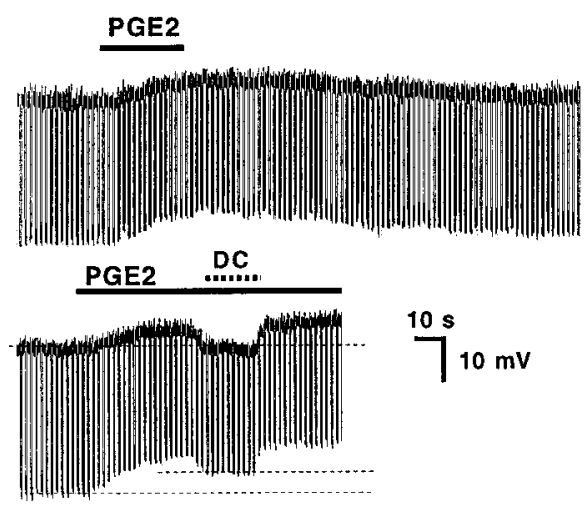

C

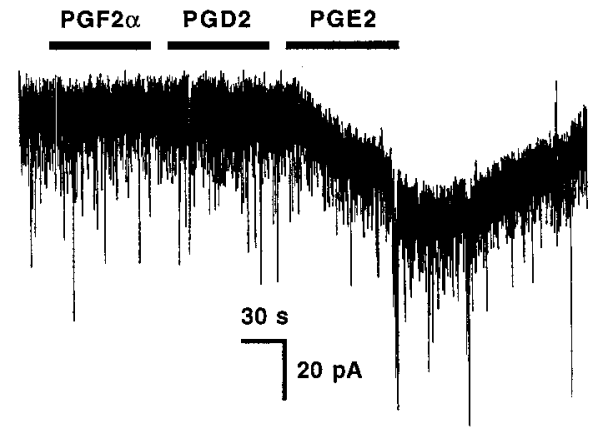

D
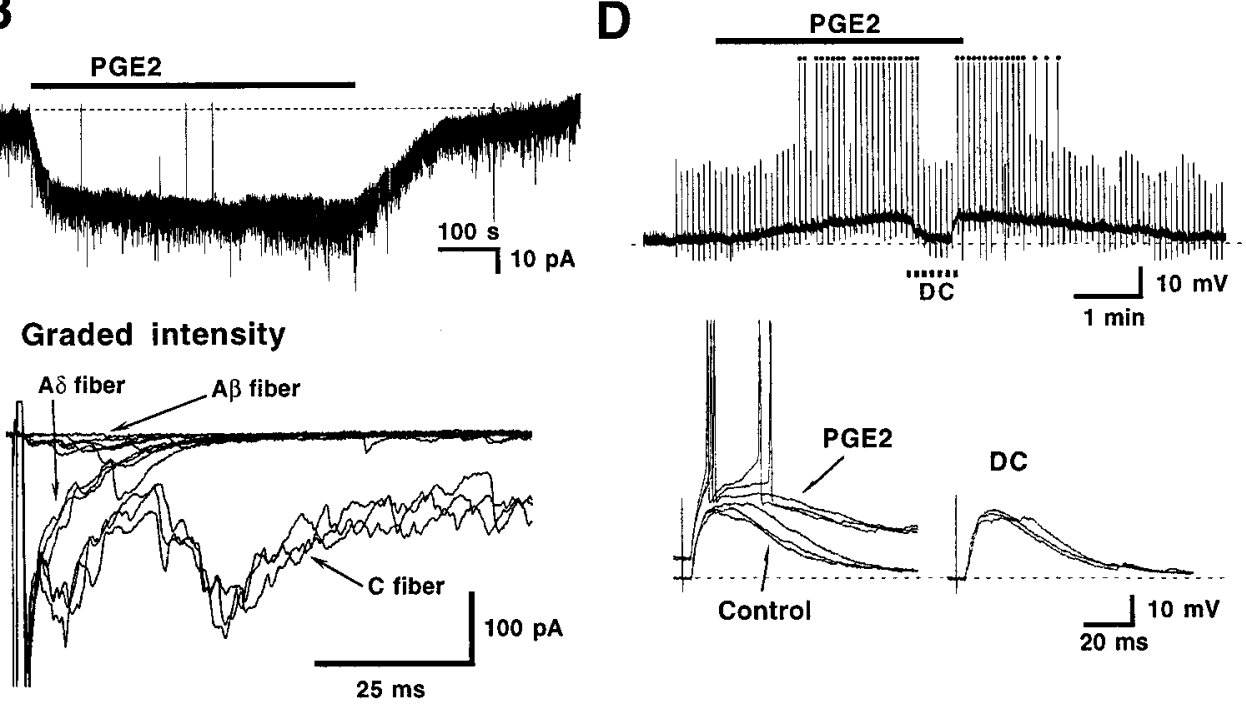

B

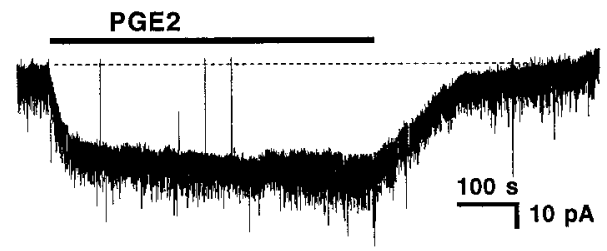

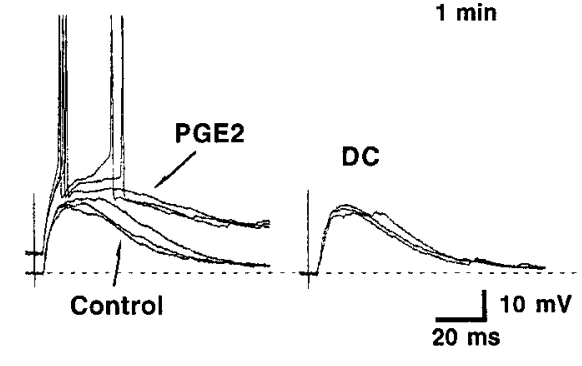

Figure 1. Effect of PGE2 on membrane properties of deep dorsal horn neurons. A, Depolarizing response produced by bath application of PGE2 (10 $\mu \mathrm{M})$ recorded intracellularly in current-clamp mode in the presence of TTX $(1 \mu \mathrm{M})$. Downward deflections are electrotonic voltage transients elicited by hyperpolarizing current pulses (amplitude, $0.15 \mathrm{nA}$; duration, $400 \mathrm{msec}$ ) to measure membrane input resistance $\left(R_{\text {in }}\right)$. The PGE2-induced depolarization was associated with a decreased $R_{\text {in }}$. When the neuron was manually clamped $(D C)$ to the resting membrane potential, the reduction in $R_{\text {in }}$ was unaffected. Therefore, the decreased $R_{\text {in }}$ was not caused by activation of voltage-gated channels. $B$, Blind whole-cell patch-clamp recording of a PGE2 $(5 \mu \mathrm{M})$-induced inward current recorded in the presence of TTX $(1 \mu \mathrm{M})$ from a neuron voltage clamped to $-70 \mathrm{mV}$. This neuron was recorded from a slice with an attached dorsal root, permitting determination of the type of afferent input. Neurons with both A- and C-fiber inputs (bottom) responded to PGE2 with larger inward currents than neurons with purely A-fiber input. $C$, Both PGF2 $\alpha(10 \mu \mathrm{M})$ and PGD2 (10 $\mu \mathrm{M})$ were without effect in this PGE2 $(10 \mu \mathrm{M})$-responsive neuron. $D$, Intracellular recording from a deep dorsal horn neuron. Top, Bath-applied PGE2 (10 $\mu \mathrm{M})$ elicited a membrane depolarization. At the resting membrane potential, dorsal root stimulation at A-fiber intensity $(30 \mu \mathrm{A}, 0.05 \mathrm{msec}, 0.5 \mathrm{~Hz})$ elicited subthreshold EPSPs. During the PGE2-induced depolarization, evoked EPSPs reached action potential threshold. Action potentials are indicated by dots. When the neuron was manually clamped $(D C)$ to the resting membrane potential, action potentials disappeared. Bottom, EPSPs and action potentials are shown on an expanded time scale. Note that the amplitudes of EPSPs during DC are not significantly different from control, indicating that the generation of action potentials is not caused by the augmentation of transmitter release from presynaptic terminals. Three consecutive traces are superimposed in each stimulating condition.

\section{MATERIALS AND METHODS}

The methods for preparing thick adult rat spinal cord slices, as well as intracellular and blind whole-cell patch-clamp recording techniques, have been described in detail previously (Yoshimura and Jessell, 1989; Yoshimura and Nishi, 1993; Baba et al., 1999). Briefly, adult male rats (7-10 weeks old; $250-350 \mathrm{gm}$ ) were anesthetized with urethane (1.5-2.0 $\mathrm{gm} / \mathrm{kg}$, i.p.), and the lumbosacral spinal cord was removed. The isolated spinal cord was then placed in preoxygenated ice-cold Krebs' solution $\left(2-4^{\circ} \mathrm{C}\right)$. After removal of the dura mater, all ventral and dorsal roots were cut, and the pia-arachnoid was removed. In some experiments, a dorsal root (L4) was preserved to permit stimulation of primary afferent fibers. The spinal cord was placed in a shallow groove formed in an agar block, glued to the bottom of a microslicer stage with cyanoacrylate adhesive, and immersed in ice-cold Krebs' solution. A 500- to 600- $\mu \mathrm{m}$ - thick transverse slice was cut on a vibrating microslicer (DTK1500; Dosaka Co. Ltd., Kyoto, Japan), placed on a nylon mesh in the recording chamber, and held in place by a titanium electron microscopy grid supported by a silver-wire loop. The slice was perfused with Krebs' solution $(15 \mathrm{ml} / \mathrm{min})$ saturated with $95 \% \mathrm{O}_{2}$ and $5 \% \mathrm{CO}_{2}$ at $36-37^{\circ} \mathrm{C}$. The Krebs' solution contained (in $\mathrm{mM}$ ): $117 \mathrm{NaCl}$, $3.6 \mathrm{KCl}, 2.5 \mathrm{CaCl}_{2}$, $1.2 \mathrm{MgCl}_{2}, 1.2 \mathrm{NaH}_{2} \mathrm{PO}_{4}, 25 \mathrm{NaHCO}_{3}$, and 11 glucose. To examine the effects of removing extracellular sodium on PGE2-induced currents, 117 $\mathrm{mm} \mathrm{NaCl}$ was replaced with $117 \mathrm{~mm} n$-methyl-D-glucamine (NMDG).

Blind whole-cell patch-clamp and intracellular techniques were used to record from dorsal horn neurons. For whole-cell patch-clamp recording, the pipette solution contained (in $\mathrm{mM}$ ): 135 potassium gluconate, $5 \mathrm{KCl}$, $0.5 \mathrm{CaCl}_{2}, 2 \mathrm{MgCl}_{2}, 5$ EGTA, 5 HEPES, 5 ATP-Mg salt, and 0.5 Na-GTP. The resistance of a typical patch pipette was 5-10 M $\Omega$. Mem- 
Table 1. PGE2 responses in dorsal horn (DH) neurons

\begin{tabular}{lll} 
& Superficial DH & Deep DH \\
\hline Lamina & II & III, IV, V, V I \\
Inward current & $5 / 37$ & $65 / 116$ \\
$\quad$ (patch clamp) & $5 \pm 2 \mathrm{pA}$ & $21 \pm 16 \mathrm{pA}$ \\
Depolarization & $1 / 16$ & $18 / 23$ \\
$\quad$ (intracellular) & $3 \mathrm{mV}$ & $8 \pm 4 \mathrm{mV}$ \\
Total & $6 / 53$ & $83 / 139$ \\
& $(11 \%)$ & $(60 \%)$ \\
\hline
\end{tabular}

brane currents (patch-clamp recording) were amplified with an Axopatch 200A amplifier (Axon Instruments, Foster City, CA) in voltage-clamp mode; usually a $75 \%$ series resistance compensation was introduced. Unless otherwise indicated, membrane currents were recorded at a holding potential of $-70 \mathrm{mV}$. For intracellular recordings, membrane potentials were amplified with an Axoclamp 2A amplifier (Axon Instruments). The resistance of a typical "sharp" electrode for intracellular recording was 150-200 $\mathrm{M} \Omega$ when filled with $4 \mathrm{M}$ potassium acetate. Signals were filtered at $2 \mathrm{kHz}$ and digitized at $5 \mathrm{kHz}$. Data were analyzed using pCLAMP 6 software (Axon Instruments).

Recording electrodes were positioned in the dorsal horn under direct visual control. In the adult spinal cord, lamina II [substantia gelatinosa (SG)] is readily identifiable as a distinct translucent region. When recording from SG neurons, the recording electrode was positioned in the middle third of the SG. We found that when an electrode is targeted to lamina II and neurons are labeled with neurobiotin, the recorded neuron is invariably located in the targeted lamina. This suggests that recording from dendrites or axons of neurons located in other laminas is extremely rare (Baba et al., 1999). The border between the SG and lamina III is clear, but the borders of laminas IV, V, and VI are not distinguishable; therefore, we dealt with laminas III-VI as one group (classified as deep dorsal horn neurons in Table 1). Blind recordings from lamina I were not attempted because of the technical difficulties that arise from the orientation of the neurons.

Orthodromic stimulation of the dorsal root (L4) was performed with a suction electrode and a constant-current stimulator (Neurolog). The threshold stimulation intensity and duration of $\mathrm{A} \beta$-fiber $(\sim 10 \mu \mathrm{A}, 0.05$ $\mathrm{msec}), \mathrm{A} \delta$-fiber $(\sim 25 \mu \mathrm{A}, 0.05 \mathrm{msec})$, and C-fiber $(\sim 200 \mu \mathrm{A}, 0.5 \mathrm{msec})$ for this suction electrode have been established previously (Baba et al.,
1999). Identification of EPSCs/EPSPs as monosynaptic was based on a constant latency with graded intensity and high-frequency repetitive stimulation $(20 \mathrm{~Hz})$. Polysynaptic EPSCs/EPSPs, in contrast, displayed variable latencies with such stimulation protocols.

Drugs were applied by exchanging the perfusion solution with one containing a known drug concentration, without altering the perfusion rate and the temperature. Drugs used were prostaglandin E2 (a gift from Ono Pharmaceutical, Osaka, Japan), flufenamic acid (FFA; Sigma, St. Louis, MO), NMDG (Sigma), 17-phenyl trinor prostaglandin E2 (Cayman Chemical, Ann Arbor, MI), sulprostone (Cayman Chemical), 19(R)-hydroxy prostaglandin E2 (Cayman Chemical), butaprost methyl ester (Cayman Chemical), and tetrodotoxin (TTX; Sigma). Prostanoid stock solutions were prepared in DMSO. Stocks were diluted 1:2800 in Krebs' solution, resulting in a final DMSO concentration of $<0.1 \%$. This concentration of DMSO produces no measurable effect on dorsal horn neurons. Data are presented as the mean \pm SD.

\section{RESULTS}

\section{PGE2-induced depolarization and inward current}

Bath-applied PGE2 (1-20 $\mu \mathrm{M})$ induced a membrane depolarization (measured by intracellular recording) or an inward current (measured by whole-cell patch-clamp recording) in most of the deep dorsal horn neurons (83 of 139 neurons in laminas III-VI) (Fig. $1 A-C$ ). In contrast, PGF2 $\alpha$ and PGD2 were without effect $(n=5$; Fig. $1 C$ ). The PGE2-induced membrane depolarization in deep dorsal horn neurons averaged $8 \pm 4 \mathrm{mV}$ (laminas III-VI; PGE2, $10 \mu \mathrm{M} ; n=18)$, whereas the inward current averaged $21 \pm$ 16 pA (laminas III-VI; PGE2, $10 \mu \mathrm{M} ; n=65$ ) (Table 1). In a subset of experiments, neurons were classified on the basis of primary afferent input (Fig. $1 B$ ). PGE2 responses were significantly greater in neurons with both $\mathrm{A}$ - and $\mathrm{C}$-fiber inputs than in neurons receiving solely A-fiber inputs $(38 \pm 23 \mathrm{pA}, n=8$, vs $16 \pm 10 \mathrm{pA}, n=9 ; p=0.02$, unpaired $t$ test). In contrast, the vast majority of lamina II neurons tested did not respond to PGE2 (10 $\mu \mathrm{M}$ ), with only 6 of 53 SG neurons (11\%) showing a PGE2induced inward current or depolarization. The amplitude of PGE2 $(10 \mu \mathrm{M})$-induced currents in those SG neurons that responded $(5 \pm 2 \mathrm{pA} ; n=5)$ was significantly smaller than in deep
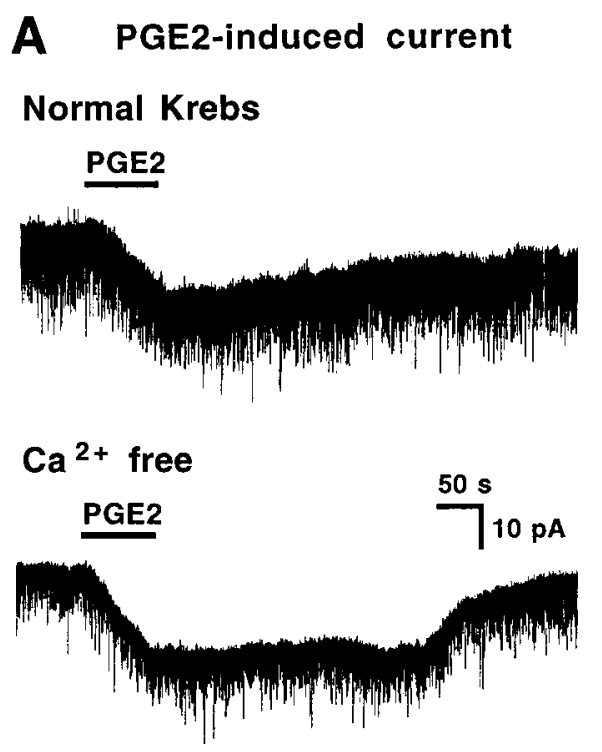

B Synaptic response Normal Krebs
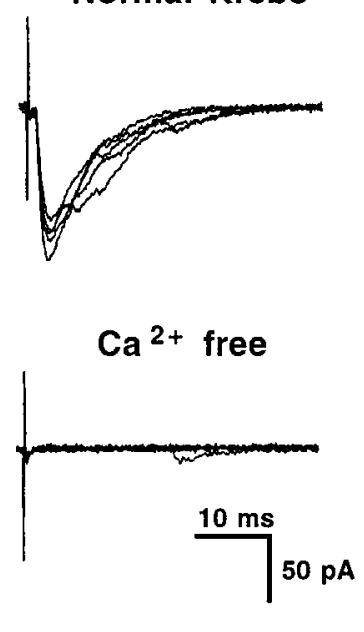

\section{C mEPSC}

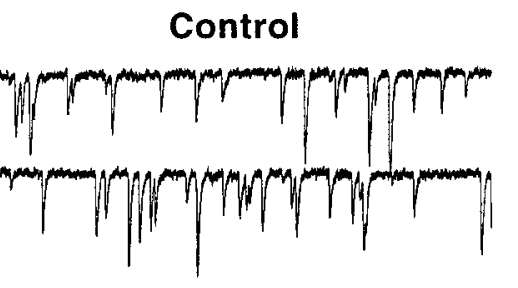

PGE2

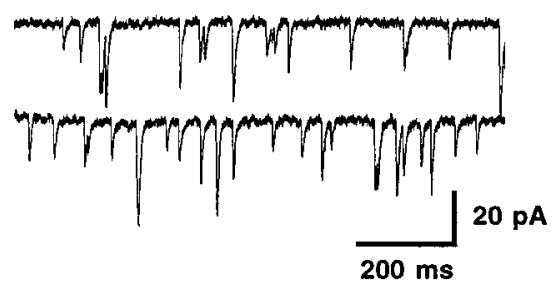

Figure 2. PGE2 acts postsynaptically. $A$, Whole-cell patch-clamp recording of a PGE2 $(5 \mu \mathrm{M})$-evoked inward current in a deep dorsal horn neuron with only A-fiber input. The PGE2-induced inward current was not blocked by perfusion with the $\mathrm{Ca}^{2+}$-free/high $\mathrm{Mg}^{2+}(5 \mathrm{mM}) \mathrm{Krebs}$ solution. $B$, In contrast, dorsal root-evoked monosynaptic EPSCs were completely abolished by perfusion with the $\mathrm{Ca}^{2+}$-free/high $\mathrm{Mg}^{2+}$ solution. Traces in $A$ and $B$ are from the same neuron. $C$, PGE2 did not affect the frequency of miniature EPSCs. The frequencies of miniature EPSCs before and during application of PGE2 were 46.2 and $44.3 \mathrm{~Hz}$, respectively. This neuron responded to PGE2 with an inward current (see Fig. 1B). 
A Control

PGE2

A $\delta$ fiber

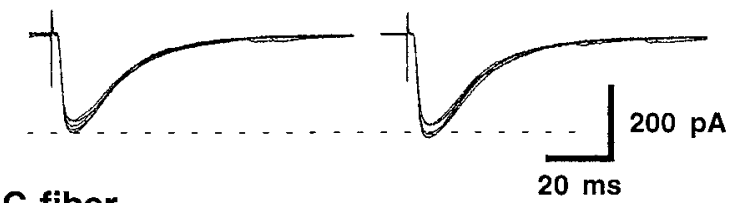

C fiber
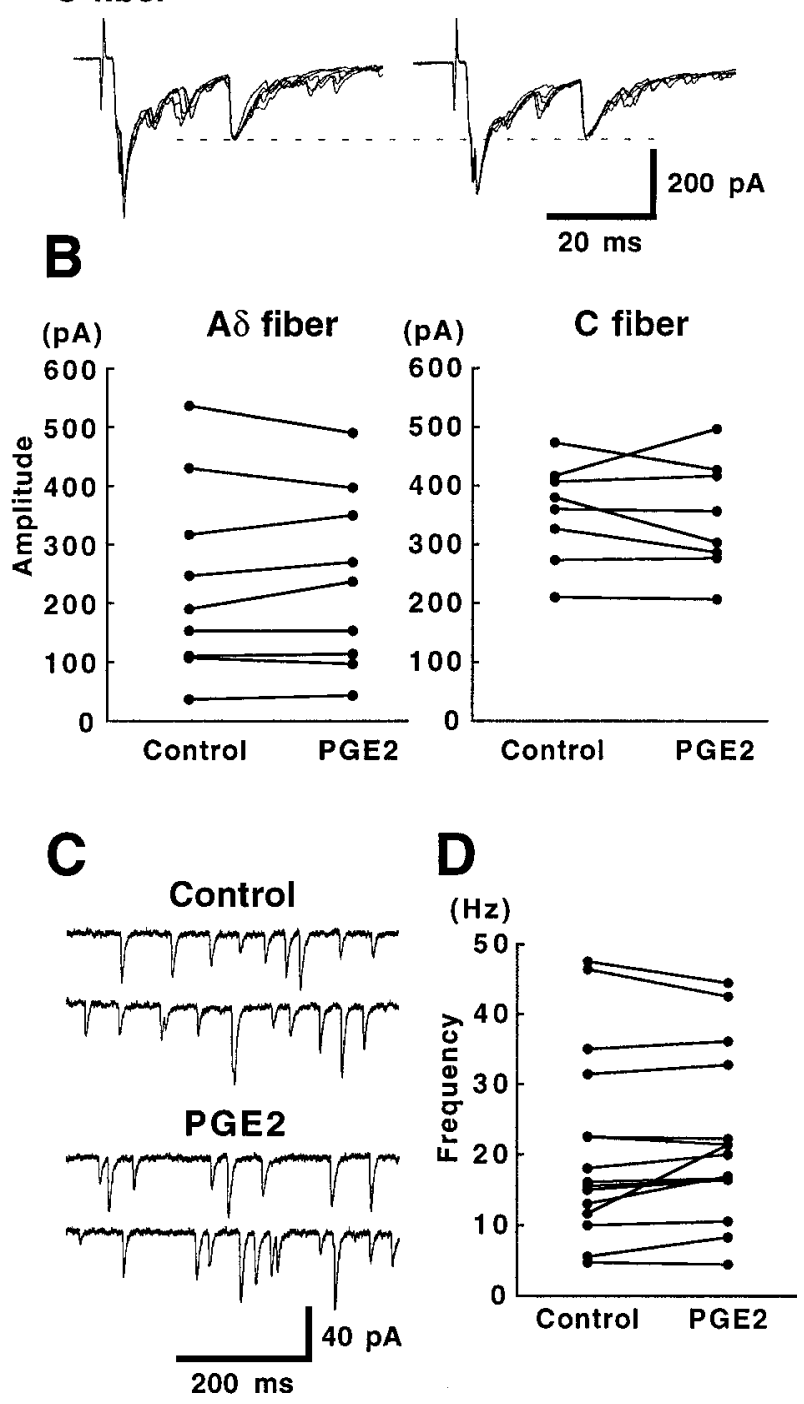

Figure 3. PGE2 has no effect on excitatory transmitter release in the SG (lamina II). $A$, The amplitude of A $\delta$-fiber (top traces) and C-fiber (bottom traces)-evoked monosynaptic EPSCs was not affected by PGE2 $(10 \mu \mathrm{M})$. Five consecutive traces are superimposed for each condition. They were averaged, and the difference between baseline and peak current was measured. $B$, Summary of the PGE2 $(10 \mu \mathrm{M})$ effects on the amplitude of dorsal root-evoked EPSCs. PGE2 did not significantly alter the amplitude of either A $\delta$ - or C-fiber-evoked EPSCs in the SG (A $\delta$-fiber EPSC, $p=$ $0.79, n=9$; C-fiber EPSC, $p=0.78, n=8$; paired $t$ test). The amplitudes of evoked EPSCs were measured 5-10 min after the start of PGE2 application. $C$, Miniature EPSCs recorded from a SG neuron in the presence of TTX $(1 \mu \mathrm{M})$. The frequencies of mEPSCs were 22.6 and 22.2 $\mathrm{Hz}$ before and during application of PGE2, respectively. $D$, The effect of PGE2 $(10 \mu \mathrm{M})$ on the frequency of mEPSCs. PGE2 did not significantly increase the frequency of mEPSCs in 14 of 15 SG neurons studied ( $p=$ 0.23 , paired $t$ test). The frequency of mEPSCs was counted 5-10 min after the start of PGE2 application. All data were recorded at $-70 \mathrm{mV}$. dorsal horn neurons $(21 \pm 16 \mathrm{pA}, n=65 ; p=0.023$, unpaired $t$ test; Table 1).

To determine whether the PGE2-induced depolarization affected the action potential generation, dorsal root-evoked responses were examined in deep dorsal horn neurons that exhibited PGE2 responses. In all six neurons tested, dorsal root stimulation at A-fiber intensity evoked either monosynaptic EPSPs or polysynaptic EPSPs, or both. Although PGE2 alone never elicited spontaneous action potentials in these neurons, it converted subthreshold inputs to suprathreshold and led to action potential generation (Fig. 1D). These data suggest that the PGE2-induced depolarization increased the probability that subthreshold EPSPs in deep dorsal horn neurons reached the action potential threshold.

Several lines of evidence suggest that PGE2 can facilitate excitatory transmitter (e.g., glutamate, substance-P, etc.) release, particularly from primary afferent C-fiber terminals (Nicol et al., 1992; Hingtgen et al., 1995; Vasko, 1995). Therefore, slow inward currents elicited by PGE2 might result from an indirect excitatory action on central primary afferent terminals (presynaptic action). To test the possibility of such an effect, the effect of PGE2 on deep dorsal horn neurons was examined either under synaptic blockade or in the presence of TTX to block action potentials. TTX $(1 \mu \mathrm{M}$; $n=10$ ) did not significantly modify the depolarizing or inward current-generating effects of PGE2 (Fig. 1A,B). Similarly, in $\mathrm{Ca}^{2+}$-free/high $\mathrm{Mg}^{2+}$ (5 mM) Krebs' solution, PGE2-induced inward currents were also fully preserved ( $n=7$; Fig. $2 A$ ), although primary afferent stimulation-evoked monosynaptic EPSCs were completely abolished (Fig. 2B). The frequency of spontaneous miniature EPSCs (mEPSCs) in those deep dorsal horn neurons that showed an inward current in response to PGE2 was also unaffected by PGE2 $(102 \pm 12 \%$ of control; $p=0.68 ; n=$ 10; paired $t$ test; Fig. 2C).

In addition, we tested whether PGE2 facilitated A $\delta$-fiber- and C-fiber-mediated excitatory transmission in the SG in which many $\mathrm{A} \delta$ - and $\mathrm{C}$-fibers make direct synaptic connections. PGE2 $(10 \mu \mathrm{M})$ had no significant effect on the amplitude of monosynaptic A $\delta$-fiber-evoked $(237 \pm 164$ vs $239 \pm 151 \mathrm{pA} ; n=9 ; p=$ $0.79)$ or C-fiber-evoked $(355 \pm 83$ vs $350 \pm 89 \mathrm{pA} ; n=8 ; p=$ 0.78) EPSCs (Fig. $3 A, B$ ). Furthermore, the frequency of miniature EPSCs in SG neurons was not significantly affected by PGE2 $(10 \mu \mathrm{M} ; n=15 ; p=0.23$; Fig. $3 C, D)$.

\section{An EP2-like receptor mediates the PGE2-induced inward current}

The known EP receptors have been divided into four subtypes, EP1, EP2, EP3, and EP4 (Coleman et al., 1994). Because selective EP receptor antagonists are not available, we used a battery of agonists to determine which EP receptor subtype mediates the PGE2-induced inward current. A nonselective EP receptor agonist, $19(R)$-hydroxy prostaglandin E2 (3-10 $\mu \mathrm{M})$ (Boie et al., 1997), and a relatively selective EP2 receptor agonist, butaprost methyl ester (3-10 $\mu \mathrm{M})$ (Boie et al., 1997), both evoked inward currents in a concentration-dependent fashion $[19(R)$-hydroxy PGE2, $17 \pm 3 \mathrm{pA}$ at $10 \mu \mathrm{M}, n=5$ of 7 ; butaprost methyl ester, $14 \pm 7 \mathrm{pA}, n=4$ of 6 ; Fig. $4 A-C]$. The $\mathrm{EC}_{50}$ of the butaprost methyl ester response was $3.1 \mu \mathrm{M}$, consistent with its binding affinity for the EP2 receptor $(2.6 \mu \mathrm{M})$ (Boie et al., 1997). In contrast, both an EP1 agonist, 17-phenyl trinor prostaglandin E2 $(0.3-10 \mu \mathrm{M} ; n=6)$, and an EP3 agonist, sulprostone $(0.3-10 \mu \mathrm{M}$; $n=5)$, had little effect in neurons that responded to PGE2 (10 $\mu \mathrm{M}$; Fig. $4 A, C)$. These results suggest that the PGE2-induced 
A

Figure 4. Effects of EP receptor agonists on membrane currents in deep dorsal horn neurons. $A$, An EP1 receptor agonist, 17-phenyl trinor prostaglandin E2 $(10 \mu \mathrm{M})$, and an EP3 receptor agonist, sulprostone $(10 \mu \mathrm{M})$, had no effect on membrane currents in neurons that responded to the nonselective EP receptor agonist, 19(R)-hydroxy prostaglandin E2 (10 $\mu \mathrm{M}) . B$, The selective EP2 receptor agonist, butaprost methyl ester $(10 \mu \mathrm{M})$, induced an inward current similar to that produced by PGE2 $(10 \mu \mathrm{M}) . C$, Comparison of the effects of the different EP agonists. Points represent the mean $\pm \mathrm{SD}$ of the percentage maximum response to PGE2 $(10 \mu \mathrm{M}) . n=3-6$ in each group. $\mathrm{EC}_{50}$ values are $1.5 \mu \mathrm{M}$ for PGE2, 2.3 $\mu \mathrm{M}$ for $19(R)$-hydroxy prostaglandin $\mathrm{E} 2$, and $3.1 \mu \mathrm{M}$ for butaprost methyl ester.

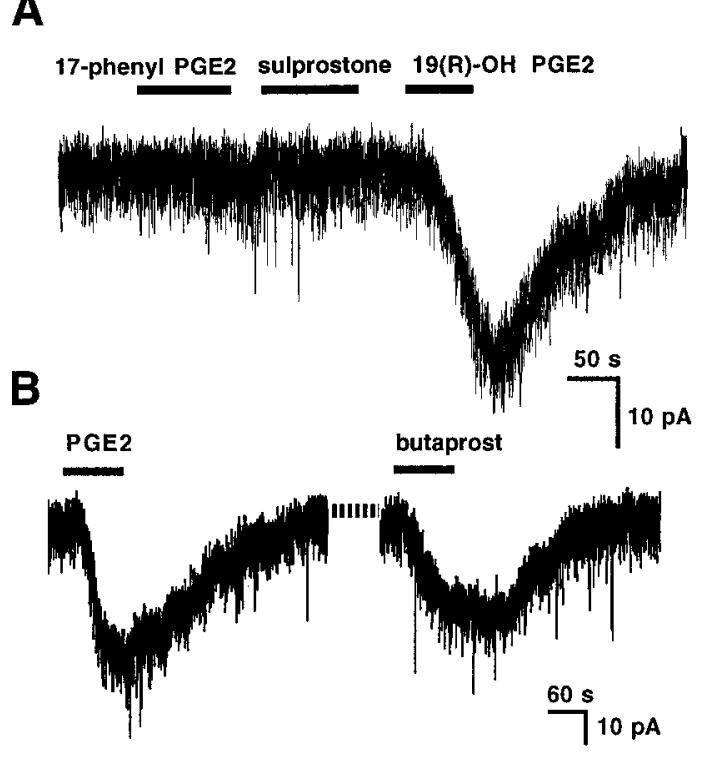

C

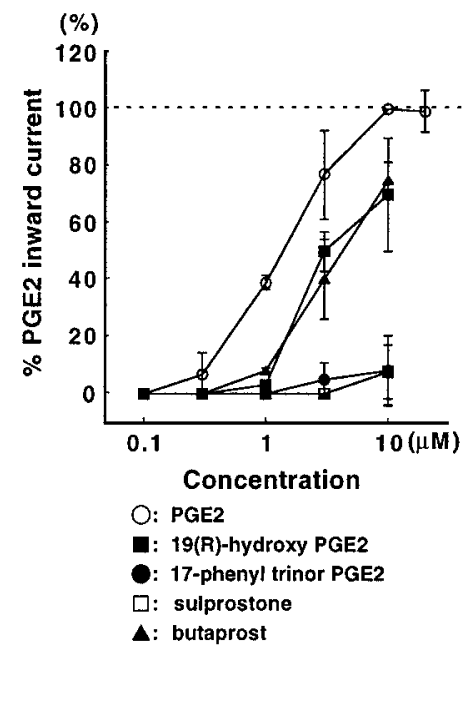

inward current is mediated by an EP receptor with an EP2-like profile.

\section{PGE2 activation of a cation conductance}

The PGE2-induced depolarization in deep dorsal horn neurons was associated with a decrease in input resistance $(13 \pm 5 \% ; n=$ 5; Fig. $1 A$ ), indicating that the opening of membrane channels, rather than the closing of potassium channels, underlies the PGE2 response. Additionally, when the holding potential was depolarized (from -90 to $-50 \mathrm{mV} ; n=4$; Fig. $5 A$ ), inward currents decreased in amplitude. Therefore, it is unlikely that activation of $\mathrm{Cl}^{-}$channels $\left(\mathrm{E}_{\mathrm{Cl}}=-70 \mathrm{mV}\right)$ contributes to the PGE2 response. In distal colon cells, PGE2 activates a nonselective cation conductance (Siemer and Gogelein, 1992). To determine whether a similar mechanism might exist in dorsal horn neurons, we examined the effects of FFA (50-200 $\mu \mathrm{M})$, a nonselective blocker of cation channels (Gogelein et al., 1990). FFA alone had no effect on membrane current, but it reversibly blocked PGE2-induced inward currents in deep dorsal horn neurons $(n=8$; Fig. $5 B, C)$. The sodium permeability of the PGE2-activated channel was also investigated by replacing extracellular sodium with a nonpermeable cation, NMDG (Fig. 5D). PGE2 was initially applied in normal Krebs' solution; then the low sodium (NMDG) solution was exchanged for normal extracellular solution. PGE2-induced inward currents were immediately reduced to nearly baseline levels by the low sodium solution in a fully reversible fashion in all cells tested $(n=4)$. Baseline membrane currents were not significantly affected by switching to the low-sodium solution.

\section{DISCUSSION}

We have found that the prostanoid PGE2 induces an inward current or membrane depolarization in many deep dorsal horn neurons from thick adult rat spinal cord slices, whereas PGF $2 \alpha$ and PGD2 have no effect. PGE2 acts directly on the recorded neurons because the inward current is preserved under conditions in which synaptic transmission is blocked. The frequency of spontaneous EPSCs is not affected by PGE2 during the inward current, further suggesting a direct postsynaptic effect. PGE2induced inward currents were associated with a reduction in input resistance, and they decreased in amplitude when the holding potential was depolarized (from -90 to $-50 \mathrm{mV}$ ). Therefore, it is unlikely that the PGE2 response is mediated by $\mathrm{K}^{+}$or $\mathrm{Cl}^{-}$ channels that have hyperpolarized equilibrium potentials $\left(\mathrm{K}^{+}=\right.$ $\left.-90 \mathrm{mV} ; \mathrm{Cl}^{-}=-70 \mathrm{mV}\right)$. Together with the reduction in the PGE2 current by flufenamic acid and the effect of replacing extracellular sodium, this suggests that the PGE2-induced depolarization may be mediated through activation of a nonselective cation channel.

Although intrathecally administered PGE2 produces allodynia and hyperalgesia (Uda et al., 1990; Minami et al., 1994), the site and mechanism of action have not yet been elucidated. Autoradiographic studies demonstrate that the most intense binding of PGE2 is in the superficial dorsal horn. Intense PGE2 binding is reduced after dorsal rhizotomy (Matsumura et al., 1995), suggesting that PGE2 binds to receptor sites on the central terminals of C-fibers. Indeed, some studies suggest that PGE2 enhances glutamate release from primary afferent $\mathrm{C}$-fiber terminals (Malmberg et al., 1995; Ferreira and Lorenzetti, 1996). PGE2 also stimulates neuropeptide release from cultured embryonic DRG neurons (Nicol et al., 1992; Vasko et al., 1994; Hingtgen et al., 1995) and spinal cord slices (Andreeva and Rang, 1993; Vasko, 1995; Southall et al., 1998). Surprisingly, in the present study, we failed to observe augmentation of identified C-fiber-evoked glutamatergic EPSCs or an increase in the frequency of miniature EPSCs. In general, neuropeptide release requires more intense excitation of presynaptic terminals than is required to elicit glutamate release. Therefore, facilitation of neuropeptide release (such as substance-P and/or calcitonin gene-related peptide) without affecting glutamate release is unlikely, unless PGE2 acts specifically to affect release from dense-core vesicles. Nonetheless, we cannot exclude a presynaptic PGE2 effect on neuropeptide-containing $\mathrm{C}$-fibers that synapse on lamina I neurons. Additional studies are required to clarify which EP receptors are located on the central terminals of primary afferents and what their role is in regulating transmitter release. What is clear is that PGE2 does exert a direct postsynaptic excitatory action on deep dorsal horn neurons.

The magnitude of the PGE2 depolarization in deep dorsal horn neurons is relatively small $(5-15 \mathrm{mV})$; although insufficient to elicit spontaneous spike firing directly, it increases the probability that primary afferent-evoked EPSPs will reach the action 


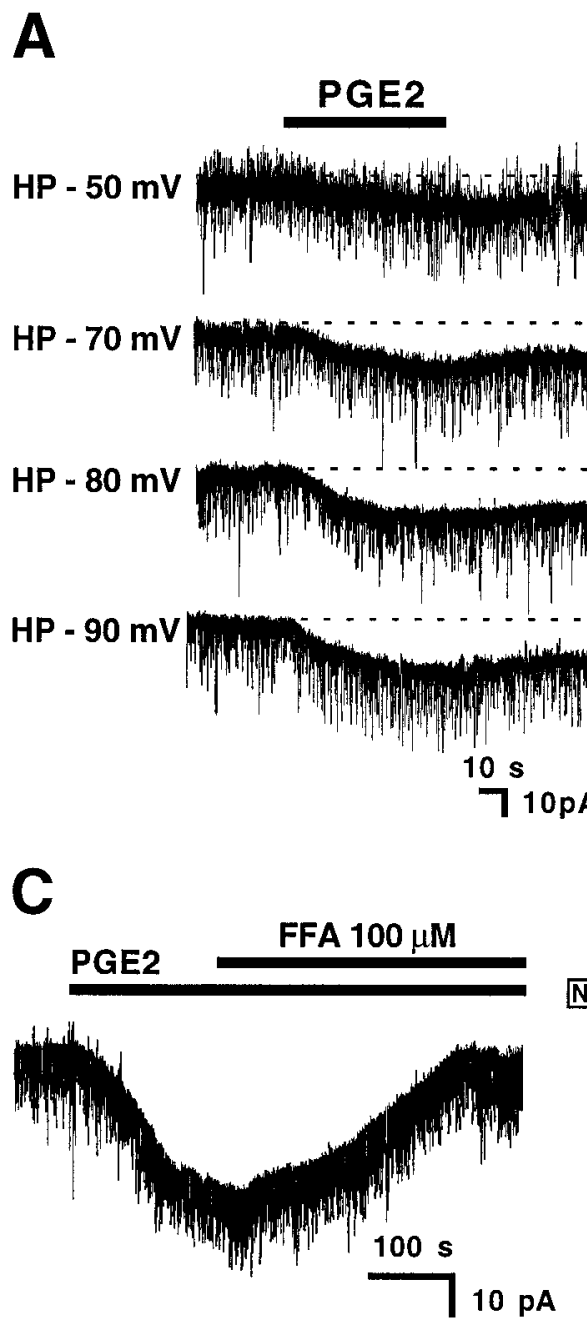

B

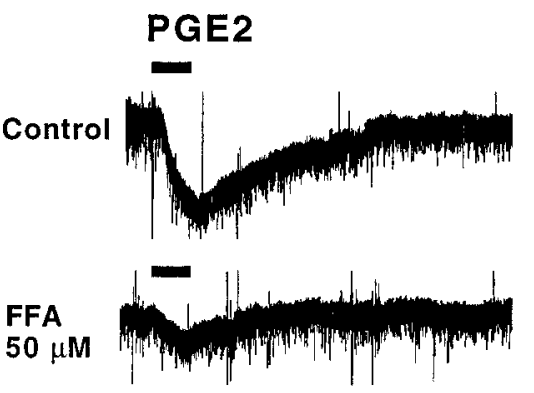

FFA $100 \mu \mathrm{M}$
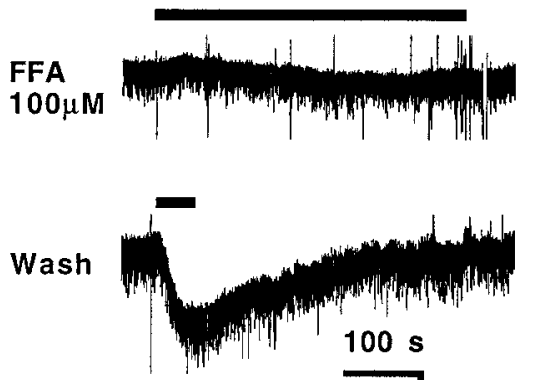

25 pA
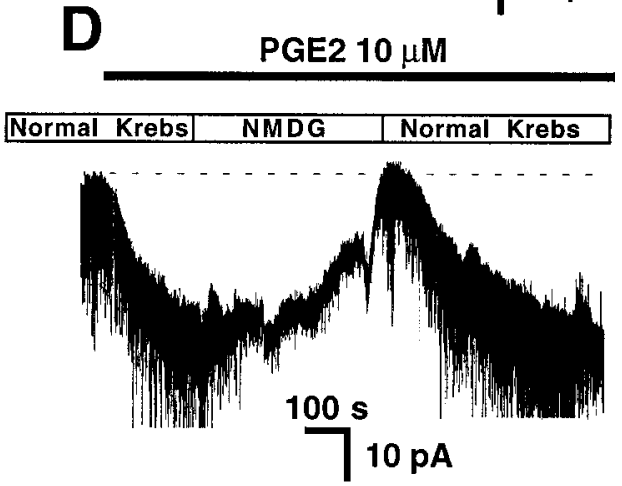

Figure 5. Effects of membrane potential, flufenamic acid $(F F A)$, and $N$-methyl-D-glucamine $(N M D G)$ on PGE2-induced inward currents. $A$, This deep dorsal horn neuron was voltage clamped to different holding potentials, and PGE2 (10 $\mu \mathrm{M}, 60 \mathrm{sec})$-induced inward currents were recorded. Note that responses become smaller as the membrane potential is depolarized. $H P$, Holding potential. $B$, Bath-applied PGE2 $(5 \mu \mathrm{M})$ elicited an inward current in another neuron, as recorded by the whole-cell patch-clamp technique. FFA $(50 \mu \mathrm{M})$ partially blocked the PGE2-induced inward current. Increasing the concentration of FFA to $100 \mu \mathrm{M}$ almost completely blocked the PGE2-induced current, in a reversible fashion. $C$, Whole-cell patch-clamp recording reveals that application of FFA $(100 \mu \mathrm{M})$ during the PGE2 $(5 \mu \mathrm{M})$-induced inward current reversed the effect of PGE2. D, Effect of decreasing extracellular $\mathrm{Na}^{+}$on PGE2induced inward currents. In normal Krebs' solution, bath-applied PGE2 (10 $\mu \mathrm{M})$ induced an inward current. Changing the perfusion solution to a low $\mathrm{Na}^{+}$solution, in which $\mathrm{Na}^{+}$was replaced with $\mathrm{NMDG}^{+}$(a membrane impermeable cation), reversed the PGE2-induced inward current nearly to control values. Returning to normal Krebs' solution restored the PGE2induced inward current. The amplitudes of spontaneous EPSCs are truncated. Neurons were voltage clamped to $-70 \mathrm{mV}$ in $B-D$. potential threshold (approximately $-45 \mathrm{mV}$ ). In this way, the PGE2-mediated depolarization could have a major role in modulating dorsal horn transmission (central sensitization; Fig. 1). Most primary afferent synaptic inputs are subthreshold, and a small increase in membrane excitability is sufficient to substantially modify receptive field properties, including increasing spatial extent and responsiveness, as well as reducing the threshold (Woolf and King, 1990). Furthermore, membrane depolarization below the action potential threshold level can generate plateau potentials via FFA-sensitive nonselective cation channels that significantly alter the input-output relationship of a proportion of deep dorsal horn neurons (Morisset and Nagy, 1999).

Determination of the EP receptor subtype involved in PGE2evoked responses has been hampered by the absence of antagonists. We have, instead, used a battery of EP receptor agonists. Although the agonists used in this study are not $100 \%$ selective for the respective EP receptors, 17-phenyl trinor PGE2 (EP1 $\gg$ EP3) and sulprostone (EP3 $\gg$ EP1) have much higher affinity for EP1 and EP3 receptors than for EP2 or EP4 receptors (Minami et al., 1994). In contrast, butaprost methyl ester is relatively selective for EP2 receptors (Coleman et al., 1994; Boie et al., 1997). We have shown that, even at high concentrations, 17phenyl trinor PGE2 and sulprostone did not mimic PGE2 action, but butaprost methyl ester did. Therefore, it is likely that the direct effects of PGE2 on deep dorsal horn neurons were mediated by an EP2-like receptor.

EP1 and EP2/EP4 receptors are coupled to $\mathrm{Ca}^{2+}$ mobilization and stimulation of adenylate cyclase (AC-cAMP-PKA cascade), respectively (Coleman et al., 1994). In contrast, depending on which splice variant is present, EP3 receptors can activate or inhibit adenylate cyclase or mobilize intracellular calcium (Narumiya et al., 1999). Interestingly, the thermal hypersensitivity induced by intrathecal PGE2 is reduced in cAMP-dependent protein kinase knock-out mice (PKA mutant mice) (Malmberg et al., 1997), in keeping with an action on EP2, EP3, or EP4 receptors. Other behavioral studies also suggest that EP2 and EP3 receptor activation elicits hyperalgesia, whereas EP1 receptor activation results in allodynia (Minami et al., 1994). Therefore, the direct depolarization of deep dorsal horn neurons described here may contribute to some of the behavioral effects of PGE2 that have been described previously.

\section{REFERENCES}

Andreeva L, Rang HP (1993) Effect of bradykinin and prostaglandins on the release of calcitonin gene-related peptide-like immunoreactivity from the rat spinal cord in vitro. Br J Pharmacol 108:185-190.

Baba H, Doubell TP, Woolf CJ (1999) Peripheral inflammation facilitates $A \beta$ fiber-mediated synaptic input to the substantia gelatinosa of the adult rat spinal cord. J Neurosci 19:859-867.

Baccaglini PI, Hogan PG (1983) Some rat sensory neurons in culture 
express characteristics of differentiated pain sensory cells. Proc Natl Acad Sci USA 80:594-598.

Beiche F, Scheuerer S, Brune K, Geisslinger G, Goppelt-Struebe M (1996) Up-regulation of cyclooxygenase-2 mRNA in the rat spinal cord following peripheral inflammation. FEBS Lett 390:165-169.

Beiche F, Brune K, Geisslinger G, Goppelt-Struebe M (1998) Expression of cyclooxygenase isoforms in the rat spinal cord and their regulation during adjuvant-induced arthritis. Inflamm Res 47:482-487.

Boie Y, Stocco R, Sawyer N, Slipetz DM, Ungrin MD, Neuschafer-Rube F, Puschel GP, Metters KM, Abramovitz M (1997) Molecular cloning and characterization of the four rat prostaglandin E2 prostanoid receptor subtypes. Eur J Pharmacol 340:227-241.

Chapman V, Dickenson AH (1992) The spinal and peripheral roles of bradykinin and prostaglandins in nociceptive processing in the rat. Eur J Pharmacol 219:427-433.

Coleman RA, Smith WL, Narumiya S (1994) International Union of Pharmacology classification of prostanoid receptors: properties, distribution, and structure of the receptors and their subtypes. Pharmacol Rev 46:205-229.

Dirig DM, Yaksh TL (1999) In vitro prostanoid release from spinal cord following peripheral inflammation: effects of substance P, NMDA and capsaicin. Br J Pharmacol 126:1333-1340.

Ferreira SH, Lorenzetti BB (1996) Intrathecal administration of prostaglandin E2 causes sensitization of the primary afferent neuron via the spinal release of glutamate. Inflamm Res 45:499-502.

Gogelein H, Dahlem D, Englert HC, Lang HJ (1990) Flufenamic acid, mefenamic acid and niflumic acid inhibit single nonselective cation channels in the rat exocrine pancreas. FEBS Lett 268:79-82.

Hingtgen CM, Waite KJ, Vasko MR (1995) Prostaglandins facilitate peptide release from rat sensory neurons by activating the adenosine 3',5'-cyclic monophosphate transduction cascade. J Neurosci 15:5411-5419.

Ichitani Y, Shi T, Haeggstrom JZ, Samuelsson B, Hokfelt T (1997) Increased levels of cyclooxygenase-2 mRNA in the rat spinal cord after peripheral inflammation: an in situ hybridization study. NeuroReport 8:2949-2952

Kumazawa T, Mizumura K, Koda H (1993) Involvement of EP3 subtype of prostaglandin E receptors in PGE2-induced enhancement of the bradykinin response of nociceptors. Brain Res 632:321-324.

Malmberg AB, Yaksh TL (1992) Antinociceptive actions of spinal nonsteroidal anti-inflammatory agents on the formalin test in the rat. J Pharmacol Exp Ther 263:136-146.

Malmberg AB, Yaksh TL (1995) Cyclooxygenase inhibition and the spinal release of prostaglandin E2 and amino acids evoked by paw formalin injection: a microdialysis study in unanesthetized rats. J Neurosci 15:2768-2776.

Malmberg AB, Hamberger A, Hedner T (1995) Effects of prostaglandin E2 and capsaicin on behavior and cerebrospinal fluid amino acid concentrations of unanesthetized rats: a microdialysis study. J Neurochem 65:2185-2193.

Malmberg AB, Brandon EP, Idzerda RL, Liu H, McKnight GS, Basbaum AI (1997) Diminished inflammation and nociceptive pain with preservation of neuropathic pain in mice with a targeted mutation of the type I regulatory subunit of cAMP-dependent protein kinase. J Neurosci 17:7462-7470.

Matsumura K, Watanabe Y, Onoe H, Watanabe Y (1995) Prostacyclin receptor in the brain and central terminals of the primary sensory neurons: an autoradiographic study using a stable prostacyclin analogue [3H]iloprost. Neuroscience 65:493-503.

Minami T, Nishihara I, Uda R, Ito S, Hyodo M, Hayaishi O (1994) Characterization of EP-receptor subtypes involved in allodynia and hyperalgesia induced by intrathecal administration of prostaglandin E2 to mice. Br J Pharmacol 112:735-740.

Morisset V, Nagy F (1999) Ionic basis for plateau potentials in deep dorsal horn neurons of the rat spinal cord. J Neurosci 19:7309-7316.

Narumiya S, Sugimoto Y, Ushikubi F (1999) Prostanoid receptors: structure, properties, and functions. Physiol Rev 79:1193-1226.

Nicol GD, Klingberg DK, Vasko MR (1992) Prostaglandin E2 increases calcium conductance and stimulates release of substance $\mathrm{P}$ in avian sensory neurons. J Neurosci 12:1917-1927.

Ohuchi K, Sato H, Tsurufuji S (1976) The content of prostaglandin E and prostaglandin F2 $\alpha$ in the exudate of carrageenin granuloma of rats. Biochim Biophys Acta 424:439-448.

Pitcher GM, Henry JL (1999) NSAID-induced cyclooxygenase inhibition differentially depresses long-lasting versus brief synapticallyelicited responses of rat spinal dorsal horn neurons in vivo. Pain 82:173-186.

Samad TA, Moore KA, Sapirstein A, Billet S, Allchorne A, Poole S, Bonventre JP, Woolf CJ (2001) An interleukin $1 \beta$-mediated induction of Cox 2 in the central nervous system contributes to inflammatory pain hypersensitivity. Nature, in press.

Siemer C, Gogelein H (1992) Activation of nonselective cation channels in the basolateral membrane of rat distal colon crypt cells by prostaglandin E2. Pflügers Arch 420:319-328.

Southall MD, Michael RL, Vasko MR (1998) Intrathecal NSAIDS attenuate inflammation-induced neuropeptide release from rat spinal cord slices. Pain 78:39-48.

Uda R, Horiguchi S, Ito S, Hyodo M, Hayaishi O (1990) Nociceptive effects induced by intrathecal administration of prostaglandin D2, E2, or F2 alpha to conscious mice. Brain Res 510:26-32.

Vasko MR (1995) Prostaglandin-induced neuropeptide release from spinal cord. Prog Brain Res 104:367-380.

Vasko MR, Campbell WB, Waite KJ (1994) Prostaglandin E2 enhances bradykinin-stimulated release of neuropeptides from rat sensory neurons in culture. J Neurosci 14:4987-4997.

Vinegar R, Truax JF, Selph JL (1976) Quantitative comparison of the analgesic and anti-inflammatory activities of aspirin, phenacetin and acetaminophen in rodents. Eur J Pharmacol 37:23-30.

Woolf CJ, King AE (1990) Dynamic alterations in the cutaneous mechanoreceptive fields of dorsal horn neurons in the rat spinal cord. J Neurosci 10:2717-2726.

Yamamoto T, Nozaki-Taguchi N (1997) Role of spinal cyclooxygenase (COX)-2 on thermal hyperalgesia evoked by carageenan injection in the rat. NeuroReport 8:2179-2182.

Yang LC, Marsala M, Yaksh TL (1996) Characterization of time course of spinal amino acids, citrulline and PGE2 release after carrageenan/ kaolin-induced knee joint inflammation: a chronic microdialysis study. Pain 67:345-354.

Yoshimura M, Jessell TM (1989) Primary afferent evoked synaptic responses and slow potential generation in rat substantia gelatinosa neurons in vitro. J Neurophysiol 62:96-108.

Yoshimura M, Nishi S (1993) Blind patch-clamp recordings from substantia gelatinosa neurons in adult rat spinal cord slices: pharmacological properties of synaptic currents. Neuroscience 53:519-526. 